16

\title{
Вклад тонких поглощающих слоев в спектры отражения
}

\author{
() А.В. Михайлов ${ }^{1}$, В.Л. Кузьмин ${ }^{2}$ \\ ${ }^{1}$ Санкт Петербургский государственный университет, \\ 198504 Санкт Петербург, Россия \\ ${ }^{2}$ Санкт Петербургский политехнический университет Петра Великого, \\ 195251 Санкт Петербург, Россия \\ e-mail: mav030655@gmail.com, kuzmin_vl@mail.ru
}

Поступила в редакцию 07.11.2019 г.

В окончательной редакции 25.11.2019 г.

Принята к публикации 02.12.2019 г.

Рассмотрена задача о вкладе, обусловленном наличием тонких поверхностных слоев, в спектры отражения. Вычислены коэффициенты отражения для двух поляризаций падающего излучения с учетом анизотропии поверхностного слоя. Продемонстрирована актуальность полученных результатов для использования в современном методе инфракрасной отражательной спектроскопии поглощения (IRRAS). Решение представляет собой обобощение развитого ранее подхода, получившего широкое распространение, на случай поглощения.

Ключевые слова: тонкие поверхностные слои, коэффициенты отражения, оптическая плотность.

DOI: $10.21883 /$ OS.2020.03.49069.301-19

\section{1. Введение}

В последнее время при исследовании границы жидкость-пар различных, представляющих прежде всего интерес для биологии и фармакологии, систем активно используется метод спектроскопии отражения с учетом поглощающих слоев в ИК области (IRRAS, infra-red reflection-absorption spectroscopy). Первые результаты были получены для нерастворимых углеводородов $[1,2]$. К настоящему времени выполнен широкий круг исследований поверхностных слоев (ПС) растворимых поверхностно-активных веществ (ПАВ), полимеров и липидов, систем, содержащих белки и ДНК, [3-15].

В указанных исследованиях толщина неоднородности на границе двух сред $L$ много меньше длины волны $\lambda$ используемого излучения, $L / \lambda \ll 1$, а коэффициенты поглощения невелики. Разработанные методики измерения позволяют регистрировать спектры оптической плотности отраженного излучения с точностью, достаточной для качественной и количественной их интерпретации. Целью исследований является получение информации о поверхностной плотности ПАВ, ориентации молекул в ПС и их зависимости от термодинамических параметров.

Практически во всех указанных работах, [3,6,7,9-14] при интерпретации результатов авторы ссылаются на наши работы по тонким пленкам [16-18], основанные на обобщении метода Друде [19] (см., например, [20]). Однако в этих работах явно эффект поглощения не рассматривался, основное внимание было уделено влиянию профиля ПС. Здесь мы излагаем теорию отражения с учетом тонкого ПС, с явным учетом поглощения, обусловленного присутствием сложных органических соединений.

Отражение на границе двух однородных сред описывается формулами Френеля. Наличие тонкого переходного слоя с эффективной толщиной, значительно меньшей длины волны, приводит к малым поправкам к формулам Френеля, известным как поправки Друде. Формально поправки первого порядка по малому параметру $L / \lambda$ являются мнимыми, поскольку обусловлены фазовым сдвигом, и наблюдаемыми являются поправки второго порядка. Однако в случае, когда поверхностные эффекты обусловлены поглощением в переходном слое, наблюдаемыми становятся вклады первого порядка, что и обусловливает возможность использования описываемого инструментария в инфракрасной области.

Метод основан на исследовании отраженного излучения, поэтому задача состоит в учете вклада ПС в коэффициенты отражения. Наиболее простой подход состоит в моделировании ПС в виде однородной пленки толщины $L$ между двумя полубесконечными средами. Для этой модели существует хорошо известное аналитическое решение, (см., например, [21]), позволяющее связать вещественную и мнимую части показателя преломления в ПС и его толщину с коэффициентами отражения поляризованного излучения. Это приближение широко применяется для численных расчетов, громоздкость окончательных выражений для коэффициентов отражения затрудняет ясное аналитическое обсуждение получаемых результатов. В работах [22-24] этот подход был упрощен в предположении $L / \lambda \ll 1$. Объектом экспериментального исследования в этих работах были пленки на электродах, что позволило сделать дополнительные упрощения вследствие больших значений мнимой части диэлектрической проницаемости металлов.

В нашем подходе также используется предположениие о малости эффективной толщины, $L / \lambda \ll 1$. В ИК области для толщин порядка $1 \mathrm{~nm}$, что соответствует большинству реальных экспериментальных ситуаций с жидкими средами, $L / \lambda \sim 10^{-3}-10^{-4}$, поэтому можно 
ожидать, что для описания эксперимента достаточно линейного приближения по $L / \lambda$. Коэффициенты отражения в этом подходе были получены в работах $[17,18]$. Полученные результаты справедливы для произвольных профилей оптических и физико-химических характеристик, включая анизотропные, поверхностные поправки выражаются в виде моментов профилей компонент тензора диэлектрической проницаемости. Коэффициенты отражения были вычислены с точностью до членов порядка $\sim(L / \lambda)^{2}$ включительно в рамках молекулярностатистического подхода. Последний позволяет не только рассчитать коэффициенты отражения, но и проанализировать связь макроскопических и молекулярных характеристик, в том числе в неоднородных системах, где понятия локальной диэлектрической проницаемости и показателя преломления нуждаются в обосновании. В этих работах рассмотрение проводилось для непоглощающих диэлектриков. В работе [18] результаты [17] были использованы для численного моделирования спектров отражения. В настоящей работе формализм, развитый в [16,17], распространен на случай поглощения в ПС и одной из сосуществующих фаз. Следует подчеркнуть, что в настоящей работе рассматривается случай внешнего отражения излучения. Важный случай НПВО рассматривался достаточно интенсивно различными авторами, например, [25,26], в которых для этого случая вычислены коэффициенты отражения как для различных профилей диэлектрической проницаемости в ПС, так и в приближении малого отличия диэлектрических проницаемостей объемной среды и ПС.

Содержание работы таково. Второй раздел содержит общую теорию отражения с учетом тонких непоглощающих неоднородных слоев; в третьем разделе получены коэффициенты отражения для неоднородных поглощающих слоев; полученные формулы непосредственно могут быть использованы для численных расчетов; четвертый раздел содержит заключение; часть выкладок перенесена в приложение.

\section{2. Отражение на границе двух сред с учетом тонких поверхностных слоев}

Пусть плоская монохроматическая световая волна падает под углом $\phi_{0}$ из среды 1 в среду 2. Рассмотрим задачу об отражении на плоской границе раздела с учетом поверхностных анизотропных неоднородностей, обусловленных ПС с произвольным профилем диэлектрической проницаемости $\widehat{\epsilon}(z)$. Состав ПС в целом не совпадает с составом прилегающих фаз, в частности, будем полагать, что молекулы ПАВ сосредоточены в ПС или отражающей среде. Для простоты положим, как и в работах [16-18], что диэлектрическая проницаемость среды 1 , из которой излучение падает на поверхность, $\epsilon_{1}=1$. Таким образом, полагаем, что вещество сосредоточено в полупространстве $z>0, z-$ координа- та, перпендикулярная плоской границе раздела, и мы ограничиваемся рассмотрением отражения на границе конденсированных сред с газом.

Распространение электромагнитной волны в среде будем описывать в терминах волнового уравнения для вектора индукции $\mathbf{D}(\mathbf{r})$ [17]:

$$
\begin{aligned}
& \mathbf{D}(\mathbf{r})=\mathbf{E}_{0}(\mathbf{r})+\int \Theta\left(z_{1}\right) \widehat{A}_{0}\left(\mathbf{r}-\mathbf{r}_{1}\right) \\
& \quad \times\left[\widehat{\psi}(z) \mathbf{D}_{2} \exp \left(i k_{2} r_{1}\right)+\Delta \mathbf{P}\left(\mathbf{r}_{1}\right)\right] d \mathbf{r}_{\mathbf{1}},
\end{aligned}
$$

где $\mathbf{E}_{0}(\mathbf{r})=\mathbf{E}_{0} \exp \left(i \mathbf{k}_{0} \mathbf{r}\right)$ - падающее поле с волновым вектором $\mathbf{k}_{0}, \mathbf{D}_{2}(\mathbf{r})=\mathbf{D}_{2} \exp \left(i \mathbf{k}_{2} \mathbf{r}\right)$ - вектор индукции в глубине среды $2, \mathbf{D}_{2}=\left(D_{2 \|} \cos \phi_{2}, D_{2 \perp},-D_{2 \|} \sin \phi_{2}\right)$, $\mathbf{k}_{2}=\left(l_{2}, 0, n_{2}\right)=k_{0} n_{2}\left(\sin \phi_{2}, 0, \cos \phi_{2}\right), n_{2}-$ волновой вектор и показатель преломления конденсированной фазы, $\mathbf{k}_{0}=\left(l_{0}, 0, m_{0}\right)=k_{0}\left(\sin \phi_{0}, 0, \cos \phi_{0}\right)-$ волновой вектор падающей плоской волны, $k_{0}=2 \pi / \lambda, \lambda$ - длина волны падающего излучения,

$$
\widehat{A}_{0}(\mathbf{r})=(\nabla \otimes \nabla-\widehat{I} \Delta) r^{-1} \exp \left(i k_{0} r\right)
$$

- функция Грина волнового уравнения для индукции, $\Theta(z)$ - функция включения Хевисайда, $\mathbf{r}=(x, y, z)-$ радиус вектор, $\Delta \mathbf{P}\left(\mathbf{r}_{\mathbf{1}}\right)$ - поверхностный избыток вектора поляризации. Множитель $\exp (-i \omega t)$, описывающий временную зависимость от частоты $\omega=c k_{0}$, опущен. Параметр

$$
\widehat{\psi}(z)=(4 \pi \widehat{\epsilon}(z))^{-1}(\widehat{\epsilon}(z)-\widehat{I})
$$

описывает профиль диэлектрической проницаемости, его зависимость от частоты принципиально важна; $\epsilon(z)=\epsilon(z \mid k)$, мы не выписываем ее для краткости обозначений.

Первое подынтегральное слагаемое дает решение в виде плоских волн и приводит, во-первых, к формулам Френеля, и во-вторых, в области ПС дает локальный вклад, отличающийся от плоской волны, который мы интерпретируем как поверхностный избыток вектора поляризации, образующий таким образом второе слагаемое.

Параметризуем задачу. Пусть волна падает в плоскости $(x, z)$. В силу трансляционной инвариантности относительно поперечных переменных, приводящей к закону Снеллиуса, поперечная компонента волнового вектора сохраняется, в том числе и в переходном слое. Решение можно представить в виде $\mathbf{D}(\mathbf{r})=\mathbf{D}(z) \exp \left(i l_{0} x\right)$.

Решение (1) ищется в виде

$$
\mathbf{D}(z)=\mathbf{D}_{2}(z)+\Delta \mathbf{D}(z)
$$

где $\mathbf{D}_{2}(\mathbf{r})$ - плоская волна в фазе $b$, а поверхностная добавка исчезает при удалении от границы, вне среды $\Delta \mathbf{D}(\mathbf{r})=0, z<0$, и внутри среды, далеко от ПС $\Delta \mathbf{D}(\mathbf{r}) \longrightarrow 0, z \longrightarrow \infty$. 
Волновое уравнение имеет вид

$$
\begin{aligned}
\mathbf{D}(\mathbf{r})+\Delta \mathbf{D}(\mathbf{r})= & \mathbf{E}_{0}(\mathbf{r})+\int \widehat{A}_{0}\left(\mathbf{r}-\mathbf{r}_{1}\right)\left[\Theta\left(z_{1}\right) \psi_{2} \mathbf{D}_{\mathbf{2}}\left(\mathbf{r}_{\mathbf{1}}\right)\right. \\
& \left.+\Delta \mathbf{P}\left(\mathbf{r}_{\mathbf{1}}\right)\right] d \mathbf{r}_{\mathbf{1}} .
\end{aligned}
$$

Мы определили здесь

$$
\Delta \mathbf{P}(\mathbf{r})=\delta \widehat{\psi}(z) \mathbf{D}_{2}(\mathbf{r})+\widehat{\psi}(z) \Delta \mathbf{D}(\mathbf{r}),
$$

где

$$
\psi_{2}=\frac{\epsilon_{2}-1}{4 \pi \epsilon_{2}}, \quad \delta \widehat{\psi}=\widehat{\psi}-\psi_{2}
$$

Первое слагаемое в (5) описывает вклад плоской волны, а второе - отклонения от набора плоских волн в поверхностный избыток вектора поляризации.

Коэффициенты отражения с учетом тонкого ПС в первом порядке по толщине являются фактически, формулами отражения Друде:

$$
\begin{aligned}
R_{p}= & R_{p}^{F}\left(1-\imath \frac{\left(m_{0}+m_{2}\right)}{\psi_{2} \cos \left(\phi_{2}+\phi_{0}\right)} \tilde{\beta}_{+}\left(m_{0}\right)\right) \\
& \times\left(1-\imath \frac{\left(m_{0}-m_{2}\right)}{\psi_{2} \cos \left(\phi_{2}-\phi_{0}\right)} \tilde{\beta}_{-}\left(-m_{0}\right)\right)^{-1}, \\
R_{s}= & R_{s}^{F}\left(1-\imath \frac{\left(m_{0}+m_{2}\right)}{\psi_{2}} \tilde{\beta}_{y}\left(m_{0}\right)\right) \\
& \times\left(1-\imath \frac{\left(m_{2}-m_{0}\right)}{\psi_{2}} \tilde{\beta}_{y}\left(-m_{0}\right)\right)^{-1},
\end{aligned}
$$

где $R_{p}^{F}$ и $R_{s}^{F}$ - коэффициенты отражения Френеля, описывающие отражение на скачкообразной границе:

$$
\begin{aligned}
& R_{p}^{F}=\left(\tan \left(\phi_{2}+\phi_{0}\right)\right)^{-1} \tan \left(\phi_{2}-\phi_{0}\right), \\
& R_{p}^{F}=\left(\sin \left(\phi_{2}+\phi_{0}\right)\right)^{-1} \sin \left(\phi_{2}-\phi_{0}\right) .
\end{aligned}
$$

Формулы (6) и (7) содержат поверхностные поправки в виде интегралов Друде (см. формулы (38)-(40) в приложении)

$$
\begin{gathered}
\tilde{\beta}_{y}\left( \pm m_{0}\right)=\int_{0}^{\infty} \frac{\epsilon_{y y}(z)-\epsilon_{2}}{4 \pi \epsilon_{2}} \exp \left( \pm ı m_{0}\right) d z, \\
\tilde{\beta}_{x}\left( \pm m_{0}\right)=\int_{0}^{\infty} \frac{\epsilon_{x x}(z)-\epsilon_{2}}{4 \pi \epsilon_{2}} \cos \phi_{2} \exp \left( \pm ı m_{0}\right) d z, \\
\tilde{\beta}_{z}\left( \pm m_{0}\right)=\int_{0}^{\infty} \frac{\epsilon_{z z}(z)-\epsilon_{2}}{4 \pi \epsilon_{2} \epsilon_{z z}(z)} \sin \phi_{2} \exp \left( \pm ı m_{0}\right) d z,
\end{gathered}
$$

где

$$
\tilde{\beta}_{ \pm}\left( \pm m_{0}\right)=\tilde{\beta}_{x}\left( \pm m_{0}\right) \cos \phi_{0} \pm \tilde{\beta}_{z}\left( \pm m_{0}\right) \sin \phi_{0} .
$$

В главном, первом порядке по толщине, аргументы интегралов Друде можно положить равными нулю. Отметим, что приведенные результаты справдливы и для среды 2 с поглощнием.

\section{3. Отражение при наличии поглощения}

Рассмотрим отражение излучения от поглощающей среды, при этом полагая, что в поверхностном слое также существует поглощение. Фактически это означает, что диэлектрические проницаемости среды 2 и ПС комплексны. Как следствие, комплексными величинами являются интегралы $I_{1}$ и $I_{2}$.

Тогда выражение для коэффициентов отражения для излучения, поляризованного в плоскости падения, $R_{p}$, и плоскости, перпендикулярной плоскости падения, $R_{s}$ имеют вид:

$$
\begin{gathered}
\tilde{R}_{s}=\tilde{R}_{s}^{F}\left(1-2\left(\tilde{\epsilon}_{2}-1\right)^{-1} \iota k_{0} \cos \phi_{0} \tilde{I}_{1}\right) \\
\tilde{R}_{p}=\tilde{R}_{p}^{F}\left(1-2\left(\tilde{\epsilon}_{2}-1\right)^{-1}\left(1-\frac{1+\tilde{\epsilon}_{2}}{\tilde{\epsilon}_{2}} \sin ^{2} \phi_{0}\right)^{-1} \imath k_{0}\right. \\
\left.\times \cos \phi_{0}\left[\tilde{I}_{1}-\left(\frac{\tilde{I}_{1}}{\tilde{\epsilon}_{2}}+\tilde{I}_{2}\right) \sin ^{2} \phi_{0}\right]\right)
\end{gathered}
$$

где

$$
\tilde{I}_{1}=\int_{0}^{\infty} d z\left(\tilde{\epsilon}_{t}(z)-\tilde{\epsilon}_{2}\right), \quad \tilde{I}_{2}=\int_{0}^{\infty} d z \frac{\tilde{\epsilon}_{n}(z)-\tilde{\epsilon}_{2}}{\tilde{\epsilon}_{n}(z)},
$$

тильда указывает на то, что величина комплексная. Коэффициенты отражения (14) и (15) позволяют вычислить эллипсометрические параметры $\Delta$ и $\Psi$ [21]:

$$
\tan \Psi \exp \left({ }_{l} \Delta\right)=\frac{\tilde{R}_{p}}{\tilde{R}_{S}} .
$$

Соотношение (17) с учетом (14) и (15) позволяет получить обобщение формулы Друде для эллипсометрических параметров в случае отражения от поглощающей среды с учетом тонкого поглощающего ПС. Для описания спектров отражения надо перейти от комплексных коэффициентов отражения (14) и (15) к энергетическим $r_{s}=\tilde{R}_{s} \tilde{R}_{s}^{*}$ и $r_{p}=\tilde{R}_{p} \tilde{R}_{p}^{*}$.

В результате перехода к вещественным параметрам получим

$$
r_{s}=r_{s}^{F}\left\{1+4 k_{0} \cos \phi_{0}\left\{\left(N_{r} I_{1 i}+N_{i} I_{1 r}\right)\right\}\right.
$$

и

$$
\begin{aligned}
& r_{p}=r_{p}^{F}\left\{1+4 k_{0} n_{1} \cos \phi_{0}\left\{Q _ { r } \left[I_{1 i}+\left(M_{i} I_{1 r}+M_{r} I_{1 i}+I_{2 i}\right)\right.\right.\right. \\
& \left.\left.\left.\quad \times \sin ^{2} \phi_{0}\right]+Q_{i}\left[I_{1 r}+\left(M_{r} I_{1 r}-M_{i} I_{1 i}+I_{2 r}\right) \sin ^{2} \phi_{0}\right]\right\}\right\},
\end{aligned}
$$

где

$$
\begin{gathered}
\tilde{N}=N_{r}+\imath N_{i}=\left(\tilde{\epsilon}_{2}-1\right)^{-1}, \quad \tilde{M}=M_{r}+\imath M_{i}=\tilde{\epsilon}_{2}^{-1}, \\
\tilde{P}=P_{r}+\imath P_{i}=\left(1-\tilde{\epsilon}_{2}^{-1}\left(1+\tilde{\epsilon}_{2}\right) \sin ^{2} \phi_{0}\right)^{-1}, \\
\tilde{Q}=Q_{r}+\imath Q_{i}=\tilde{N} \tilde{P}=N_{r} P_{r}-N_{i} P_{i}+\imath\left(N_{r} P_{i}+N_{i} P_{r}\right) .
\end{gathered}
$$

Выражения (18) и (19) являются точными энергетическими коэффициентами в первом порядке по $L / \lambda$. 
В принципе выражения для $\tilde{N}, \tilde{M}, \tilde{P}, \tilde{Q}$ в определенных ситуациях могут позволить определенные упрощения, например, если поглощение в среде 2 или в ПС мало.

Выражая введенные параметры через компоненты профиля диэлектрической проницаемости, коэффициенты отражения для интенсивности (18) и (19) представим в виде

$$
r_{s}=r_{s}^{F}\left\{1+4 k_{0} \cos \phi_{0} \frac{\left(\epsilon_{2}^{\prime}-1\right) I_{1 i}-\epsilon_{2}^{\prime \prime} I_{1 r}}{\left(\epsilon_{2}^{\prime}-1\right)^{2}+\epsilon_{2}^{\prime \prime 2}}\right\}
$$

Выражение для коэффициента отражения излучения, поляризованного в плоскости, перпендикулярной плоскости падения, к сожалению, значительно более громоздко:

$$
\begin{aligned}
& r_{p}=r_{p}^{F}\left\{1+4 k_{0} \cos \phi_{0}\right. \\
& \left.\times \frac{\left(\epsilon_{2}^{\prime}-1\right) I_{1 i}-\epsilon_{2}^{\prime \prime} I_{1 r}+I_{s 2} \sin ^{2} \phi_{0}+I_{s 4} \sin ^{4} \phi_{0}}{\left[\left(\epsilon_{2}^{\prime}-1\right)^{2}+\epsilon_{2}^{\prime \prime 2}\right]\left[\left(1-O_{r} \sin ^{2} \phi_{0}\right)^{2}+O_{i}^{2} \sin ^{4} \phi_{0}\right]}\right\},
\end{aligned}
$$

где для краткости определены параметры, представляющие собой комбинации компенент диэлектрической проницаемости:

$$
\begin{gathered}
O_{r}=\left(\epsilon_{2}^{\prime 2}+\epsilon_{2}^{\prime \prime 2}\right)^{-1}\left(\epsilon_{2}^{\prime}\left(\epsilon_{2}^{\prime}+1\right)+\epsilon_{2}^{\prime \prime 2}\right), \\
O_{i}=-\left(\epsilon_{2}^{\prime 2}+\epsilon_{2}^{\prime \prime 2}\right)^{-1} \epsilon_{2}^{\prime \prime} .
\end{gathered}
$$

В случае $\epsilon_{1}=1$, для однородного и изотропного ПС формула (20) совпадает с приведенной в [24].

Для использования в численных расчетах приведем результаты в вещественных переменных, отдельно объединяя комбинации интегралов Друде с заданной угловой зависимостью:

$$
\begin{aligned}
I_{s 2}=- & \left(\left(\epsilon_{2}^{\prime}-1\right) I_{1 i}-\epsilon_{2}^{\prime \prime} I_{1 r}\right)-\left(\left(\epsilon_{2}^{\prime}-1\right) I_{2 i}-\epsilon_{2}^{\prime \prime} I_{2 r}\right) \\
- & 2 \frac{\left(\epsilon_{2}^{\prime}-1\right) I_{1 i}-\epsilon_{2}^{\prime \prime} I_{1 r}}{\epsilon_{2}^{\prime 2}+\epsilon_{2}^{\prime \prime 2}} \epsilon_{2}^{\prime} \\
I_{s 4}= & \epsilon_{2}^{\prime} I_{2 i}-\epsilon_{2}^{\prime \prime} I_{2 r}-\frac{\epsilon_{2}^{\prime} I_{2 i}+\epsilon_{2}^{\prime \prime} I_{2 r}}{\epsilon_{2}^{\prime 2}+\epsilon_{2}^{\prime \prime 2}} \\
& +\frac{\epsilon_{2}^{\prime 2}-\epsilon_{2}^{\prime \prime 2}-1}{\epsilon_{2}^{\prime 2}+\epsilon_{2}^{\prime \prime 2}} I_{1 i}-2 \frac{\epsilon_{2}^{\prime} \epsilon_{2}^{\prime \prime}}{\epsilon_{2}^{\prime 2}+\epsilon_{2}^{\prime \prime 2}} I_{1 r}
\end{aligned}
$$

Из уравнений (21)-(23) видно, что при $\phi_{1} \rightarrow 0 r_{p} \rightarrow r_{s}$. Введя обозначения

$$
I_{1}^{-}=\left(\epsilon_{2}^{\prime}-1\right) I_{1 i}-\epsilon_{2}^{\prime \prime} I_{1 r}, \quad I_{2}^{-}=\left(\epsilon_{2}^{\prime}-1\right) I_{2 i}-\epsilon_{2}^{\prime \prime} I_{2 r},
$$

можно представить $I_{s 2}$ в виде

$$
I_{s 2}=-\left(1+2 \frac{\epsilon_{2}^{\prime}}{\epsilon_{2}^{\prime 2}+\epsilon_{2}^{\prime \prime 2}}\right) I_{1}^{-}-I_{2}^{-}
$$

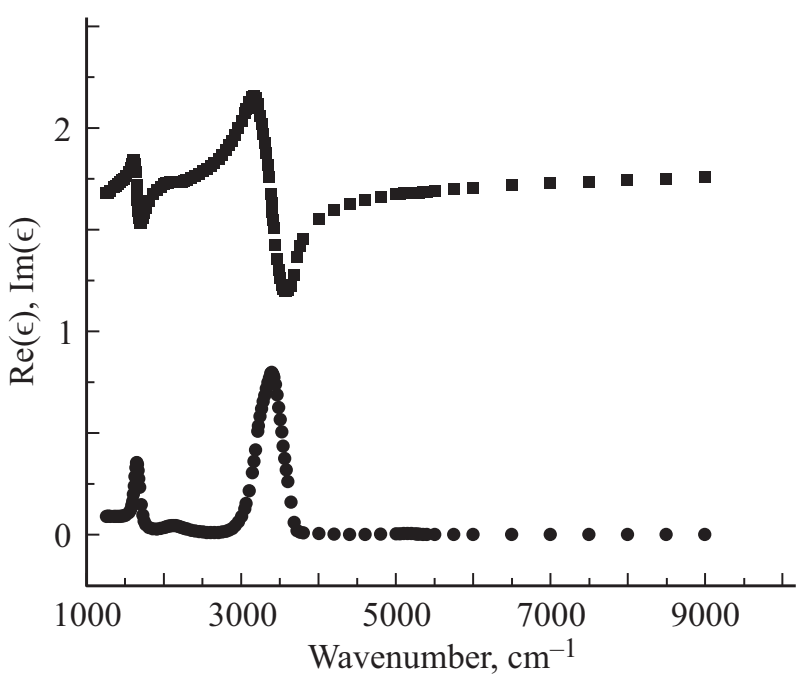

Зависимость вещественной (квадраты) и мнимой (круги) частей диэлектрической проницаемости воды от волнового числа.

Выражение для оптической плотности излучения, поляризованного перпендикулярно плоскости и в плоскости падения, на основе (20) и (21) можно представить в виде

$$
a_{s}=-\frac{4 k_{0} \cos \phi_{0}}{\ln 10} \frac{I_{1}^{-}}{\left(\epsilon_{2}^{\prime}-1\right)^{2}+\epsilon_{2}^{\prime 2}}
$$

$$
\begin{aligned}
& a_{p}=\frac{a_{s}}{\left(1-O_{r} \sin ^{2} \phi_{0}\right)^{2}+O_{i}^{2} \sin ^{4} \phi_{0}}-\frac{4 k_{0} \cos \phi_{0}}{\ln (10)} \\
& \times \frac{I_{s 2} \sin ^{2} \phi_{0}+I_{s 4} \sin ^{4} \phi_{0}}{\left[\left(\epsilon_{2}^{\prime}-1\right)^{2}+\epsilon_{2}^{\prime \prime 2}\right]\left[\left(1-O_{r} \sin ^{2} \phi_{0}\right)^{2}+O_{i}^{2} \sin ^{4} \phi_{0}\right]},
\end{aligned}
$$

где $a_{s}=-\lg \left(r_{s} / r_{s}^{F}\right), a_{p}=-\lg \left(r_{p} / r_{p}^{F}\right)$.

В работе [18] выражение (25) достаточно подробно обсуждалось в предположении $\epsilon_{2}^{\prime \prime} \ll \epsilon_{2}^{\prime}-1$. Чтобы обсудить ограничения, налагаемые этим приближением, приведем данные для вещественной и мнимой частей диэлектрической проницаемости воды [27], которые используются большинством исследователей, интерпретирующих спектры отражения от поверхности воды или водных растворов.

Из рисунка видно, что в обсуждаемой в [18] области $2800-3000 \mathrm{~cm}^{-1}$ мнимая часть диэлектрической проницаемости много меньше вещественной, и ею можно пренебречь. В областях как валентных, так и деформационных колебаний молекул воды, $\epsilon_{2}^{\prime \prime} \backsim \epsilon_{2}^{\prime}-1$, и, таким образом, здесь пренебрежение членами $\sim \epsilon_{2}^{\prime \prime}$ неоправдано. Следует подчеркнуть, что учет этого члена существен в области волновых чисел $1600-1700 \mathrm{~cm}^{-1}$, исключительно важной при обсуждении структуры ПС белков на поверхности воды или водных растворов. 


\section{4. Заключение}

Таким образом, мы получили выражения для коэффициентов отражения и оптической плотности отраженного поляризованного излучения с учетом вклада тонкого анизотропного ПС. Для поляризации в плоскости, перпендикулярной плоскости падения, результат весьма компактен. Для поляризации в плоскости падения, как и следовало ожидать, результат более громоздок, но даже в этом случае анализ экспериментальных данных не требует сложной численной обработки, что обеспечивает прозрачность интерпретации экспериментальных данных. Укажем на простую угловую зависимость этой компоненты отражения, позволяющей дополнительный количественный анализ данных измерений. Полученные результаты окажутся полезными при моделировании поверхностных профилей, и в особенности моделировании ориентации сложных поверхностно-активных молекул в ПС. Следует подчеркнуть, что хотя почти вся процитированная в работе литература относится к исследованиям в ИК диапазоне, развитый формализм равно применим и к видимому, и УФ диапазонам. Естественно, что поскольку в этом случае длины волн значительно меньше, то сокращается и дипазон толщин ПС, для которых справедливо приближение их малости по сравнению с $\lambda$.

\section{5. Приложение}

Представим функцию Грина волнового уравнения в виде фурье-интеграла

$$
\widehat{A}_{0}(\mathbf{r})=4 \pi(\nabla \otimes \nabla-\widehat{I} \Delta) \int \frac{d \mathbf{q}}{(2 \pi)^{3}} \frac{\exp (\iota \mathbf{q r})}{q^{2}-\left(k_{0}+\imath \eta\right)^{2}} .
$$

Подставляя (27) в интегральный член волнового уравнения и выполняя интегрирование по поперечным переменным с учетом трансляционной инвариантности, получим

$$
\begin{aligned}
& \widehat{\mathbf{I}}(\mathbf{r})=\int \widehat{A_{0}}\left(\mathbf{r}-\mathbf{r}_{1}\right) \widehat{\psi}\left(\mathbf{r}_{1}\right) \mathbf{D}\left(\mathbf{r}_{1}\right) d \mathbf{r}_{1} \\
& =4 \pi(\nabla \otimes \nabla-\widehat{I} \Delta) \exp (l l x) \int_{-\infty}^{\infty} d z_{1} \widehat{\psi}\left(z_{1}\right) \mathbf{D}\left(z_{1}\right) \\
& \times \int_{-\infty}^{\infty} \frac{d q_{z}}{2 \pi} \frac{\exp \left(l\left(z-z_{1}\right) m_{0}\right)}{q_{z}{ }^{2}+l^{2}-\left(k_{0}+\imath \eta\right)^{2}-l^{2}}
\end{aligned}
$$

Закон Снеллиуса обусловливает инвариантную зависимость от переменноой $x$. Интеграл Фурье по $q_{z}$ выполняется на основе теоремы о вычетах:

$$
\begin{aligned}
& \widehat{\mathbf{I}}(\mathbf{r})=\frac{2 \pi \imath}{m_{0}}(\nabla \otimes \nabla-\widehat{I} \Delta) \exp (i l x) \int_{-\infty}^{\infty} d z_{1} \\
& \quad \times \exp \left(\iota\left|z-z_{1}\right| m_{0}\right)\left(\Theta\left(z_{1}\right) \psi(z) \mathbf{D}_{2}\left(z_{1}\right)+\Delta \mathbf{P}\left(z_{1}\right)\right) .
\end{aligned}
$$

Нетрудно видеть, что в силу условия поперечности волнового вектора $\mathbf{k}_{l}$ и соответствующего поля $\mathbf{D}_{l} \exp \left(i \mathbf{k}_{l} \mathbf{r}\right), \mathbf{D}_{l} \mathbf{k}_{l}=0$, лежащего в плоскости падения, оператор $\nabla \times \nabla-\hat{I} \Delta$, действуя на любой вектор $\mathbf{A}$, представляет собой проектор на поле $\mathbf{D}_{l}$ :

$$
(\nabla \times \nabla-\hat{I} \Delta) \exp \left(i \mathbf{k}_{l} \mathbf{r}\right) \mathbf{A}=k_{l}^{2} \exp \left(i \mathbf{k}_{l} \mathbf{r}\right) \mathbf{A} D_{l}^{-2} \mathbf{D}_{l}\left(\mathbf{D}_{l} \mathbf{k}_{l}\right),
$$

для любого из полей $\mathbf{D}_{l}$ : падающего, $\mathbf{D}_{0}=\mathbf{E}_{0}$, преломленного, $\mathbf{D}_{2}$, или отраженного $\mathbf{D}^{\prime}=\mathbf{E}^{\prime}$.

Вычислим первое слагаемое (29)

$$
\begin{aligned}
\mathbf{I}_{0}= & \frac{2 \pi \imath}{m_{0}}(\nabla \otimes \nabla-\widehat{I} \Delta) \\
& \times \exp (i l x) \int_{0}^{\infty} d z_{1} \exp \left(l m_{0}\left|z-z_{1}\right|+m_{2} z_{1}\right) \psi_{0} \mathbf{D}_{2}
\end{aligned}
$$

для случаев $z<0$ и $z>0$. $\mathbf{I}_{0}$ имеет вид

$$
\mathbf{I}_{0}=\frac{2 \pi \psi_{2}}{m_{0}\left(m_{2}+m_{0}\right)} \mathbf{k}_{0}^{\prime} \times \mathbf{k}_{0}^{\prime} \times \mathbf{D}_{2}
$$

при $z<0$, и

$$
\mathbf{I}_{0}=\frac{4 \pi \psi_{2}}{\left(m_{0}^{2}-m_{2}^{2}\right)} \mathbf{k}_{2} \times \mathbf{k}_{2} \times \mathbf{D}_{2}+\frac{2 \pi \psi_{2}}{m_{0}\left(m_{2}-m_{0}\right)} \mathbf{k}_{0} \times \mathbf{k}_{0} \times \mathbf{D}_{2}
$$

при $z>0$. Находя отношение амплитуд отностительно амплитуды падающей волны, разрешая уравнение (1) с учетом (31) и (32), т.е. фактически в предположении $\Delta \mathbf{P}(z)=0$, получим формулы Френеля для отраженной $(8),(9)$ и преломленной волн.

Интеграл от второго слагаемого в (29) в общем виде не вычисляется, так как требуется конкретный вид профиля.

С учетом члена, обусловленного поверхностным избытком вектора поляризации, получим для полей падающей и отраженной волн

$$
\begin{aligned}
\mathbf{E}^{\prime}= & \frac{2 \pi \psi_{2}}{m_{0}\left(m_{2}+m_{0}\right)} \mathbf{k}_{0}^{\prime} \times \mathbf{k}_{0}^{\prime} \times\left[\mathbf{D}_{2}-\imath \frac{m_{2}+m_{0}}{\psi_{2}}\right. \\
& \left.\times \int_{0}^{\infty} d z_{1} \Delta \mathbf{P}\left(z_{1}\right) \exp \left(\imath m_{0} z_{1}\right)\right], \\
\mathbf{E}_{0}= & -\frac{2 \pi \psi_{0}}{m_{0}\left(m_{2}-m_{0}\right)} \mathbf{k}_{0} \times \mathbf{k}_{0} \times\left[\mathbf{D}_{2}-\imath \frac{m_{2}-m_{0}}{\psi_{2}}\right. \\
& \left.\times \int_{0}^{\infty} d z_{1} \Delta \mathbf{P}\left(z_{1}\right) \exp \left(-\imath m_{0} z_{1}\right)\right] .
\end{aligned}
$$

Выражение для отклонения от набора плоских волн в ПС имеет вид

$$
\begin{aligned}
& \Delta \mathbf{D}\left(z_{1}\right)=4 \pi\left[\Delta \mathbf{P}\left(z_{1}\right)-\mathbf{f} \otimes \mathbf{f} \Delta \mathbf{P}\left(z_{1}\right)\right] \\
& -\frac{2 \pi \imath}{m_{0}} \int_{z_{1}}^{\infty} d z_{2}\left[\mathbf{k}_{0}^{\prime} \times \mathbf{k}_{0}^{\prime} \times \exp \left(\iota m_{0}\left(z_{2}-z_{1}\right)\right)\right. \\
& \left.-\mathbf{k}_{0} \times \mathbf{k}_{0} \times \exp \left(-\imath m_{0}\left(z_{2}-z_{1}\right)\right)\right] \Delta \mathbf{P}\left(z_{2}\right) .
\end{aligned}
$$

где $\mathbf{f}$ - орт вдоль оси $z$. 
Рассмотрим решение в главном порядке по параметру $L / \lambda$. В этом случае достаточно ограничиться первым слагаемым в (35). Тогда выражение для $\Delta \mathbf{D}(z)$ можно записать как

$$
\Delta \mathbf{D}(\mathbf{z})=4 \pi(\widehat{I}-\mathbf{f} \otimes \mathbf{f}) \Delta \mathbf{P}(z)
$$

а выражение для поверхностного избытка поляризации (5) имеет вид

$$
\Delta \mathbf{P}(z)=\delta \hat{\psi}(z) \mathbf{D}_{2}(z)+4 \pi \hat{\psi}(z)(\widehat{I}-\mathbf{f} \times \mathbf{f}) \Delta \mathbf{P}(z)
$$

или

$$
\Delta \mathbf{P}(z)=(1-4 \pi \hat{\psi}(z)(1-\mathbf{f} \times \mathbf{f}))^{-1} \delta \hat{\psi}(z) \mathbf{D}_{2}(z) .
$$

В проекциях

$$
\begin{gathered}
\Delta \mathbf{P}_{x}=\epsilon_{x x}(z) \delta \psi_{x x}(z) D_{b x}=D_{2 \|} \frac{\epsilon_{x x}(z)-\epsilon_{2}}{4 \pi \epsilon_{2}} \cos \phi_{2} \\
\Delta \mathbf{P}_{y}=\epsilon_{y y}(z) \delta \psi_{y y}(z) D_{b y}=D_{2 \perp} \frac{\epsilon_{y y}(z)-\epsilon_{2}}{4 \pi \epsilon_{2}} \\
\Delta \mathbf{P}_{z}=\delta \psi_{z z}(z) D_{b z}=-D_{2 \|} \frac{\epsilon_{z z}(z)-\epsilon_{2}}{4 \pi \epsilon_{2} \epsilon_{z z}(z)} \sin \phi_{2}
\end{gathered}
$$

\section{Список литературы}

[1] Dluhy R., Cornell D. // J. Phys. Chem. 1985. V. 89. P. 3195.

[2] Dluhy R. // J. Phys. Chem. 1986. V. 90. P. 1373.

[3] Mendelson R., Mao G., Flach C.R. // Biochimica et Biophysica Acta. 2010. V. 1798. P. 788.

[4] Briggs D., Cornell R., Dluhy R., Gierasch L. // Science. 1986. V. 233. P. 206.

[5] Dluhy R., Reilly K., Hunt R., Mitchell M., Mautone A., Mendelson R. // Biophys. J. 1989. V. 56. P. 1173.

[6] Bi X., Taneva S., Keough K., Mendelson R., Flach C. // Biochemistry. 2001. V. 40. P. 13659.

[7] Du X., Miao W., Liang Y. // J. Phys. Chem. B. 2005. V. 109. P. 7428.

[8] Wang L., Brauner J., Mao G., Crouch E., Seaton E., Head J., Smith K., Flach C., Mendelson R. // Biochemistry. 2008. V. 47. P. 8103.

[9] Zheng H., Du X. // J. Phys. Chem. B. 2010. V. 114. P. 557.

[10] Wang X., Huang X., Xin Y., Du X. // Phys. Chem. Chem. Phys. 2012. V. 14. P. 5470.

[11] Stefaniu C., Brezesinski G., Moewald H. // Advances in Colloid and Interface Science. 2014. V. 208. P. 197.

[12] Rabe M., Schwieger C., Zope H., Versluis F., Kros A. // Langmuir. 2014. V. 30. P. 7724.

[13] Schwieger C., Liub X., Krafft M.P. // Phys. Chem. Chem. Phys. 2017. V. 19. P. 23809.

[14] Koepf E., Richert M., Braunschweig B., Schroeder R., Brezesinski G., Friessa W. // Int. J. of Pharmaceutics. 2018. V. 541. P. 234.

[15] Lyadinskaya V., Lin Shi-Yow, Michailov A., Povolotskiy A., Noskov B. // Langmuir. 2016. V. 32. P. 13435.

[16] Kuzmin V., Mikhailov A. // Opt. Spectrosc. 1981. V. 51. P. 383.

[17] Kuzmin V., Romanov V., Mikhailov A. // Opt. Spectrosc. 1992. V. 73. P. 1.
[18] Gericke A., Michailov A., Huhnerfuss H. // Vib. Spectrosc. 1993. V. 4. P. 335.

[19] Drude P. The Theory of Optics. Dover Publications Inc. 1959.

[20] Kuzmin V.L., Romanov V.P., Zubkov L.A. Phys. Rep. 1994. V. 248. P. 71.

[21] Azzam R.M.A., Bashara N.M. Ellipsometry and Polarized Light. North-Holland Pub. Co., 1977. 529 p.

[22] Mcintire J., Aspens D. // Surf. Sci. 1971. V. 24. P. 417.

[23] Kolb D., Mcintire J. // Surf. Sci. 1971. V. 28. P. 321.

[24] Mcintire J. // Surf. Sci. 1973. V. 37. P. 658.

[25] Розанов Н.Н., Золотарев В.М. // Опт. и спектр. 1980. Т. 49. C. 925.

[26] Мансуров Г.М., Розанов Н.Н., Золотарев В.М., Сутовский С.М. // Опт. и спектр. 1982. Т. 53. С. 301.

[27] Bertie J., Ahmed M., Eysel H. // J. Phys. Chem. 1989. V. 93. P. 2210. 